\section{Cahiers de Narratologie}

Analyse et théorie narratives

$28 \mid 2015$

Le récit comme acte cognitif

\title{
Narratologie et sciences cognitives : une relation problématique*
}

\section{Marie-Laure Ryan}

\section{(2) OpenEdition}

\section{Journals}

Electronic version

URL: https://journals.openedition.org/narratologie/7171

DOI: 10.4000/narratologie. 7171

ISSN: 1765-307X

\section{Publisher}

LIRCES

\section{Electronic reference}

Marie-Laure Ryan, "Narratologie et sciences cognitives : une relation problématique*", Cahiers de Narratologie [Online], 28 | 2015, Online since 29 October 2015, connection on 11 June 2021. URL: http://journals.openedition.org/narratologie/7171 ; DOI: https://doi.org/10.4000/narratologie.7171

This text was automatically generated on 11 June 2021.

Article L.111-1 du Code de la propriété intellectuelle. 


\title{
Narratologie et sciences cognitives : une relation problématique*
}

\author{
Marie-Laure Ryan
}

\section{AUTHOR'S NOTE}

*Cet article est une traduction fortement révisée d'un article paru dans Style 44, No. 4 (Winter 2010), pp. 469-95.

1 À première vue, mon titre peut paraître sacrilège, ou du moins totalement ignorant des tendances récentes en narratologie. Le fonctionnement de l'esprit humain ${ }^{1}$ - objet des sciences dites cognitives-n'est-il pas l'un des sujets les plus brûlants de la recherche scientifique, et l'un de ceux qui connaissent le développement le plus rapide? La narration (et son produit le récit) n'est-elle pas reconnue comme une activité qui donne un sens à notre être-dans-le-monde, à nos relations interpersonnelles, à la temporalité de notre existence, et qui par conséquent met en jeu des mécanismes cognitifs de la plus haute importance ? Les approches inspirées par les sciences cognitives ne représentent-elles pas la vague de l'avenir pour les études littéraires («the hot new thing») et leur meilleure chance de survie dans une culture dominée par la science et la technologie, comme le proclame un article paru dans le New York Times (Cohen 2010)?

Mais en dépit de l'intérêt croissant pour le rôle de la narration dans la formation de l'intelligence et la vie de l'esprit, l'importance des contributions concrètes des sciences cognitives à la narratologie sont loin d'entraîner l'unanimité des chercheurs. Pour démontrer la nature problématique des relations entre ces deux domaines, je voudrais prendre à témoin un article datant de 2009 intitulé "La scanographie du cerveau suggère que les lecteurs construisent de vivantes simulations mentales des situations narratives» (« Readers build vivid mental simulations of narrative situations, brain scans suggest »). Écrit par un journaliste, Gerry Everding, et publié par le service des relations publiques de Washington University à Saint Louis, cet article a pour but de 
faire connaitre la recherche de la faculté de ladite université. Voici quelques-uns des points principaux de l'article :

Une nouvelle étude d'imagerie cérébrale contribue à expliquer ce que nous voulons dire par « se perdre dans un bon livre ». Cette étude suggère que les lecteurs créent de vivantes simulations mentales des sons, des gestes, des goûts et des mouvements décrits dans un récit écrit, et qu'au cours de ces simulations les mêmes régions du cerveau sont activées que lors d'expériences similaires dans la vie quotidienne.

« Les psychologues et neurologistes en viennent de plus en plus à la conclusion que quand nous lisons une histoire et la comprenons véritablement, nous créons une simulation mentale des événements décrits par le texte", déclare l'un des coauteurs de l'étude, Jeffrey M. Zacks, Ph.D., professeur associé de psychologie en Arts et Sciences et de Radiologie à l'école de médecine, ainsi que directeur du Laboratoire de cognition dynamique dans le département de psychologie.

Nicole Speer, Ph.D., auteur principale de l'étude, déclare que les résultats démontrent que la lecture n'est en aucun cas un exercice passif. Bien au contraire, les lecteurs produisent des simulations mentales de chacune des nouvelles situations rencontrées dans un récit. Le lecteur capte à partir du texte des détails sur les actions et les sensations des personnages et les intègre à une connaissance personnelle bâtie sur ses expériences passées. Ces données sont ensuite livrées à un mécanisme de simulation mentale basé sur des régions du cerveau qui correspondent étroitement aux régions stimulées quand les gens exécutent, imaginent ou observent des activités similaires dans le monde réel.

Voici ce qu'on a découvert. Les changements dans les objets qu'un personnage manipule (par exemple, "tirer un cordon électrique») sont associés à des augmentations d'activité dans une région des lobes frontaux connue pour contrôler les mouvements de préhension.

Les changements dans la situation spatiale des personnages (par exemple, «traverser la porte d'entrée et entrer dans la cuisine») sont associés à des augmentations d'activité dans les régions des lobes temporaux qui sont sélectivement stimulées quand on voit des images de scènes spatiales. (Everding, en ligne.)

3 Ma première réaction quand j'ai lu cet article a été le sentiment de satisfaction (bien connu des mères) qu'on exprime par la phrase «Je vous l'avais bien dit ». J'ai traité du phénomène de l'immersion dans mon ouvrage Narrative as Virtual Reality (2001), et pour décrire et expliquer ce phénomène j'ai emprunté à la psychologie cognitive la notion de simulation mentale (Oatley 1999). Cette notion peut être associée à un autre concept proposé par le psychologue Rolf Zwaan, celui de modèle de situation («situation model»), terme qui désigne l'image du monde narratif que les lecteurs (ou spectateurs) ${ }^{2}$ construisent au fil du déroulement de l'histoire, et qu'ils mettent constamment à jour pour intégrer les changements d'état décrits par le texte, mais sans perdre de vue les états précédents. Selon cette théorie, suivre une histoire revient à construire l'histoire du monde dans lequel elle se déroule. À première vue, l'expérience décrite dans l'article apporte une vérification scientifique au phénomène de la modélisation du récit. Les images IRM ${ }^{3}$ de Zacks et Speer prouvent que la simulation mentale existe réellement ! Hourra!

4 À la réflexion, cependant, mon enthousiasme s'est considérablement atténué. Comment Zacks et Speer (ou plutôt le journaliste qui leur sert de porte-parole) saventils que les lecteurs construisent de vivantes simulations mentales, et non pas des images vagues? Comment peut-on mesurer la quantité d'information contemplée par l'œil intérieur de l'imagination? Selon les témoignages recueillis par le psychologue Victor Nell, le degré de précision des représentations mentales varie considérablement selon le tempérament et l'intérêt du lecteur : certaines personnes construisent une 
image détaillée du visage d'Emma Bovary (en s'inspirant peut-être d'un film ou d'illustrations), alors que d'autres ne se représentent qu'un corps fantomatique se déplaçant à travers un paysage. Et sur quoi se base l'auteur de l'article quand il affirme que les lecteurs simulent le goût ainsi que la vue, le son et le mouvement? Comme l'observe G. Gabrielle Starr, il est beaucoup plus difficile d'imaginer des sensations olfactives et gustatives que des sons, des spectacles et des mouvements : il se pourrait très bien qu'au lieu de les simuler (c'est-à-dire de les percevoir en imagination), nous traitions les références au goût et à l'odorat « sémantiquement ", ce qui veut dire sur la base de la signification des mots et de leurs connotations (2010:285).

5 L'article scientifique qui constitue la source de l'article de publicité (Speer et al., 2009) propose une interprétation beaucoup plus mesurée. Au lieu de parler de «vive simulation mentale » Speer et son collègue décrivent deux résultats dans un langage sobrement scientifique :

6 1. La lecture d'un événement stimule les mêmes régions du cerveau que l'expérience directe de cet événement. Les sciences neurologiques sont capables de créer une carte rudimentaire du cerveau; par exemple, nous savons que l'expérience spatiale est localisée dans une certaine région, et l'expérience de la manipulation d'un objet dans une autre. Les expériences menées par Zacks et Speer nous disent que les mêmes régions sont stimulées quand nous lisons que le héros d'une histoire se déplace à l'intérieur d'une maison ou saisit un objet, et lorsque nous effectuons les mêmes activités. Les expériences menées par d'autres chercheurs avec des sujets qui lisent des mots isolés, ou avec des chimpanzés qui observent et imitent un comportement, avaient déjà produit de tels résultats, qu'on explique généralement par la postulation d'un système de neurones miroirs chez l'homme aussi bien que chez les singes ${ }^{4}$. Speer et ses collègues ont toutefois été les premiers à soumettre à leurs sujets un récit continu. Ils ont utilisé une histoire pour enfants très simple et l'ont présentée à leurs sujets un mot à la fois, afin d'enregistrer les états du cerveau à des moments précis. Ce recours à un texte narratif plutôt qu'à des mots isolés mène à la deuxième conclusion :

$7 \quad 2$. Lorsque les sujets lisent des mots isolés leur cerveau produit des signaux différents que quand ils les lisent comme faisant partie d'une histoire. En outre, certaines régions du cerveau ne sont activées que quand l'état de l'univers narratif subit de multiples changements d'une phrase à l'autre. Cela suggère que la simulation mentale nécessite un effort plus intense.

$8 \quad$ Mais ces idées ne vont-elles pas de soi ? Avons-nous besoin d'une IRM pour nous dire que la lecture de mots isolés ne nécessite pas la même activité mentale que la lecture d'une histoire cohérente? Faut-il la technologie d'imagerie du cerveau pour nous apprendre qu'il y a quelque chose de commun entre accomplir et observer une action, ou entre appréhender l'image d'un objet, cette image soit-elle verbale ou visuelle, et appréhender son référent? Comparez, par exemple, l'expérience d'être attaqué par un méchant chien par rapport à la lecture d'une histoire relatant une telle attaque. S'il n'y avait pas une activité cérébrale commune aux deux expériences (et par conséquent une certaine configuration de neurones actifs dans les deux cas), comment le lecteur pourrait-il établir une relation entre elles ? Comment pourrait-il utiliser son expérience et ses souvenirs personnels pour combler les blancs dans l'histoire ? Dans une nouvelle intitulée "Funès ou la mémoire ", Jorge Luis Borges décrit ce qui se passerait si l'idée de chien n'activait pas une idée commune pour l'ensemble de ses manifestations : nous aurions des images mentales complètement séparées non seulement pour les 
chihuahuas et les rottweilers, mais aussi pour les chiens jaunes et les chiens féroces, pour les chiens à 4 heures de l'après-midi et les chiens en terre cuite. Notre cerveau serait par conséquent incapable de les relier les unes aux autres. La découverte (ou l'hypothèse) d'un système de neurones miroirs a fait sensation dans les sciences humaines; mais que fait-elle d'autre que de produire une explication physiologique pour des phénomènes accessibles à l'intuition? Au lieu d'ouvrir de nouvelles perspectives sur l'activité cognitive propre au récit, les expériences de Zacks et Speer confirment ainsi ce que nous savons déjà. L'importance des expériences scientifiques est traditionnellement considérée comme inversement proportionnelle à la prévisibilité des résultats. Mais les techniques actuelles d'imagerie cérébrale n'ont pas encore atteint la précision nécessaire pour produire des perspectives vraiment nouvelles et intéressantes sur les fondements cognitifs de la narration. D'un point de vue narratologique, les expériences d'imagerie du cerveau sont dans une situation difficile : si elles contredisent la théorie dominante, elles sont hérétiques ; mais si elles la confirment pleinement, elles sont superflues.

9 Il y a au moins deux raisons pour lesquelles les sciences cognitives « dures » (par là je veux dire celles qui reposent sur une technologie avancée) ne peuvent pas vraiment montrer ce qui se passe dans notre tête quand nous créons ou décodons des récits. La première de ces raisons est la complexité du réseau de neurones et synapses du cerveau. Ce réseau comprend 100 milliards de neurones, chacun relié à d'autres neurones par plus de 10000 synapses. L'imagerie IRM est actuellement incapable de donner une image exacte du cerveau neurone par neurone; tout ce qu'elle peut faire est indiquer quelles régions du cerveau produisent une activité électrique sous divers stimuli. L'IRM peut être capable de détecter que lire le mot "chien» et regarder un chien activent une région du cerveau spécialisée dans la visualisation, mais il n'est pas (encore) capable d'identifier une configuration de neurones actifs qui signifient «chien» pour un certain sujet. Si les techniques d'imagerie deviennent un jour en mesure de le faire (elles s'en rapprochent de plus en plus), elles seront capables de lire les pensées, une possibilité aux conséquences effrayantes 5 .

10 La seconde, et à mon avis la plus grave limitation de la démarche scientifique dure dérive de ce que les philosophes appellent le problème de la relation de l'esprit et du corps («mind-body problem»). Ce problème consiste à expliquer comment la conscience, un phénomène spirituel, peut émerger du cerveau, un organe fait de matière purement physique. La position dominante dans les sciences cognitives et la philosophie de l'esprit est le rejet de toute explication qui postule un dualisme (dit cartésien) de l'esprit et du corps, et l'adoption d'une position matérialiste qui considère l'esprit comme le résultat de l'activité électrique du cerveau. Cette activité produit divers états du cerveau, ce qui veut dire différentes configurations de neurones à charge positive et négative. Mais la position matérialiste est aussi incapable que la position dualiste de résoudre le problème des relations du corps et de l'esprit. Alors que la position dualiste ne peut pas expliquer comment un phénomène mental comme l'intention peut causer des événements physiques, comme presser la gâchette d'un pistolet, la position matérialiste ne peut pas expliquer comment la conscience émerge de certains états du cerveau, et comment ces états peuvent produire des idées. Comme Douglas Hofstadter le remarque, il y a toujours un écart béant entre le niveau des neurones et le niveau des symboles. La distinction entre le niveau des neurones et le niveau des symboles explique pourquoi l'IRM est d'une utilité limitée pour l'étude de l'activité cognitive associée à la narration: en tant que mode de signification, le récit 
implique un niveau cognitif dont les éléments de base sont des symboles et non des neurones.

11 Je n'ai certainement pas l'intention de nier l'intérêt scientifique d'expériences comme celles de Zacks et Speer, ni de décourager ce type de recherches; elles jouent un rôle important dans la cartographie du cerveau, un projet d'une importance capitale pour la compréhension du fonctionnement de l'esprit. Mon but est plutôt de mettre en question l'importance de ces travaux pour la narratologie, au moins dans l'état actuel du développement de l'imagerie cérébrale. En d'autres termes, je voudrais poser la question de ce que j'ai à apprendre, en tant que narratologue, de ce genre de recherche. Vais-je poursuivre avec plus d'enthousiasme l'étude du phénomène d'immersion narrative, maintenant que l'imagerie cérébrale me dit que la simulation mentale existe réellement? Ou vais-je considérer ce problème comme résolu, maintenant que la science a sanctionné une réponse particulière ? Ce dilemme souligne le vaste problème des relations entre les disciplines spéculatives, comme la philosophie et la narratologie, et les disciplines expérimentales, telles que la neuroscience et la psychologie cognitive. Que faut-il faire pour que les disciplines spéculatives et expérimentales s'enrichissent mutuellement? Dans quelles conditions la coopération interdisciplinaire est-elle possible - en narratologie tout aussi bien que dans d'autres domaines?

12 La science cognitive ne se limite pas à des expériences avec l'IRM. Le terme générique de «science cognitive» recouvre une variété d'approches provenant de diverses disciplines : la neurologie, l'intelligence artificielle, la linguistique, différents types de psychologie (évolutionniste, sociale et cognitive), et la philosophie de l'esprit. Ces études de l'activité mentale diffèrent les unes des autres par l'importance relative de l'expérimentation et de la spéculation: la recherche neurologique représente le pôle expérimental, et la philosophie de l'esprit le pôle spéculatif'.

13 Les narratologues qui pratiquent l'approche cognitive, tels que Richard Gerrig et David Herman, déplorent le fait que dans leur domaine, toute influence va dans un sens unique : des sciences cognitives à la narratologie et non l'inverse (Herman $2013: 203$ ). Cette situation est-elle inévitable ou bien faut-il l'attribuer à l'état encore primitif de la narratologie cognitive? Cette question reflète la nature problématique de la narratologie cognitive, un projet pris en sandwich entre les disciplines spéculatives et interprétatives des sciences humaines et les disciplines expérimentales des sciences naturelles.

14 Si nous traçons un axe qui mène de l'interprétation à la vérification expérimentale, l'extrême gauche (j'emploie gauche et droite sans implications politiques) sera occupée par la critique littéraire, une discipline traditionnellement concernée par le sens et la qualité esthétique de textes individuels. Un pur critique littéraire est un super-lecteur, un virtuose de l'interprétation qui conçoit les textes comme des messages encryptés, et qui les décode par un déploiement de style, d'érudition et de perspicacité, tout comme un violoniste transforme en musique une partition de par sa technique et sa sensibilité. Le critère de réussite pour le critique littéraire est de révéler des significations auxquelles personne n'a pensé auparavant. Le but de la critique littéraire de l'époque postmoderne n'est donc pas de produire un savoir objectif sur les textes, mais plutôt de jouer avec eux de manière créative.

15 À droite de la critique littéraire se situe la narratologie classique (je considère la narratologie cognitive comme étant post-classique). Cette narratologie ne met pas l'accent sur l'interprétation, mais sur la description, la comparaison et la classification. 
Elle ne s'intéresse pas aux caractéristiques individuelles des textes, mais aux traits qui pourraient apparaître dans une variété de textes. Par exemple, la narratologie regarde le récit à la deuxième personne (comme on la trouve dans La Modification de Michel Butor) moins comme une expression particulière de subjectivité que comme une possibilité narrative qui complète le paradigme linguistique de la conjugaison verbale. En fait, il n'importe pas vraiment qu'un certain dispositif narratif soit utilisé par un auteur: pour être digne d'attention, il suffit qu'il soit possible. Alors que la phase classique de la narratologie était surtout préoccupée par la fiction littéraire, et ne différait de la critique littéraire que par son intérêt pour le général au lieu du particulier, dans sa phase post-classique elle conçoit l'histoire comme un type de signification qui peut se manifester à travers une pluralité de médias et de genres de discours.

16 Laissant provisoirement une place vide pour la narratologie cognitive, passons vers la droite avec la psychologie expérimentale, fondée par des chercheurs comme Walter Kintsch, David Rummelhard, Jean Mandler et Nancy Johnson, Nancy Stein and Tom Trabasso, et pratiquées de nos jours par Richard Gerrig, Catherine Emmott, and Marisa Bortolussi and Peter Dixon, pour ne nommer que quelques-uns. Tout comme la critique littéraire, la psychologie expérimentale vise à l'interprétation. Mais l'analogie s'arrête là : alors que la critique littéraire cherche à développer des lectures hautement subtiles et individualistes, la psychologie expérimentale s'intéresse aux opérations mentales les plus automatiques, celles que chaque lecteur effectue inconsciemment. Ces opérations sont souvent impossibles à distinguer de la compréhension du langage (Gerrig et Egidi 2003). Par exemple, la psychologie expérimentale se demandera : comment les lecteurs parviennent à identifier les référents des pronoms; quel est l'élément de la structure du récit dont les lecteurs se souviennent le plus facilement; dans quelle mesure les lecteurs gardent à l'esprit les buts des personnages quand le texte raconte les événements qui interrompent la poursuite de ces buts, et comment les lecteurs relient l'information récemment acquise à l'information stockée dans la mémoire à long terme. Aussi bien la critique littéraire que la narratologie classique considèrent ces opérations comme trop banales et trop automatiques pour être dignes d'attention. Une autre différence importante entre les disciplines de gauche et de droite réside dans leur centre d'intérêt. Les approches psychologiques du récit ne s'intéressent ni aux textes individuels, ni à la classe de tous les récits, mais à la nature de la compréhension. En d'autres termes, leur préoccupation est l'esprit. C'est pourquoi les psychologues n'ont aucun scrupule à utiliser des textes artificiels spécialement conçus pour tester leurs hypothèses, plutôt que des textes dignes d'être lus pour eux-mêmes.

17 Si les approches de gauche se concentrent sur les textes et les approches de droite sur l'esprit lui-même, on peut se demander s'il reste quelque chose entre ces deux pôles qui puisse faire l'objet de la narratologie cognitive. Cette école est actuellement un bricolage interdisciplinaire qui emprunte des idées à droite et à gauche, combinant librement l'approche de bas en haut («bottom-up») typique de la narratologie classique et post-classique, une approche qui forge ses propres outils, avec une application de haut en bas ("top-down») de concepts empruntés aux sciences cognitives dures. Mais cet emprunt de concepts scientifiques ne va pas jusqu'à l'adoption des rigueurs de l'expérimentation. Les travaux qui passent comme exemples de narratologie cognitive restent strictement spéculatifs, et certains narratologues fascinés par l'activité mentale nécessaire à la compréhension des récits se déclarent totalement désintéressés par les approches expérimentales. En outre, contrairement 
aux versions expérimentales des sciences de l'esprit, la narratologie cognitive ne veut pas complètement sacrifier l'intérêt pour le texte, même si elle traite souvent les œuvres comme un prétexte ou guide (cf. l'expression de «tutor text » fréquente chez Herman) pour la démonstration d'idées empruntées à la droite.

18 L'activité cognitive relative à la narration peut être étudiée dans trois domaines :

1. L'activité mentale des personnages.

2. L'activité mentale du lecteur.

3. La narration comme mode de pensée (ou : l'intelligence narrative, l'importance de la narration dans la vie de l'esprit).

\section{L'activité mentale des personnages}

19 L'activité mentale des personnages n'a pas attendu le développement de la narratologie cognitive pour susciter l'intérêt des chercheurs. La critique littéraire traditionnelle, sous l'influence de la psychanalyse, regarde l'esprit comme le site d'une activité secrète et souvent inconsciente, faite de rêves, de désirs, d'obsessions et d'illusions. La narratologie classique ajoute à ces thèmes un catalogue des techniques narratives à travers lesquelles l'activité mentale peut être représentée dans son intériorité : monologue intérieur, psychonarration, discours direct et indirect, et leur hybride, le discours indirect libre (Cohn, Banfield). Cette approche est très productive dans la description de la dimension privée de l'esprit, mais comme Alan Palmer (2004) l'a observé, elle ignore ses manifestations publiques, ainsi que ses dimensions interpersonnelles. Inspiré par les théories dites enactivistes, Herman (2011) va jusqu'à déclarer que l'esprit est tout entier dirigé vers le monde extérieur, ce qui le rend lisible pour autrui ; il s'ensuivrait qu'il n'y a pas de différence fondamentale entre les esprits fictifs (« fictional minds») et réels, contrairement à la position de narratologues tels que Cohn qui considère la transparence des pensées comme l'un des traits distinctifs de la fiction. Ma position personnelle est que la question de la transparence est fallacieuse. Les pensées privées des personnages de fiction ne sont accessibles que pour le narrateur impersonnel extradiégétique (dit pour cette raison omniscient), qui n'a pas les propriétés d'un être humain. Dans leurs relations interpersonnelles, par contre, les personnages de fiction sont ni plus ni moins transparents les uns aux autres que les êtres réels, à moins qu'il ne s'agisse de personnages surnaturels. Il s'ensuit que la transparence n'est pas une propriété intrinsèque des personnages de fiction, et qu'il n'y a pas d'autre différence ontologique entre les personnages d'un roman réaliste et les êtres réels que celle d'habiter des mondes différents (ou de ne pas exister). La différence entre fiction et non-fiction en ce qui concerne l'activité mentale se situe au niveau du mode de représentation, et non au niveau des facultés des personnages ${ }^{7}$.

20 Il n'en reste pas moins que le récit est une forme de représentation axée sur l'action humaine, et que c'est par leurs actions, bien plus que par la description de leurs pensées intérieures ou de leurs émotions, que les personnages révèlent leur état d'esprit. Pour comprendre l'action humaine, et par conséquent pour comprendre une intrigue, il est nécessaire de construire les désirs, les buts, les croyances et les plans qui motivent les agents.

21 La plupart des bons récits gardent les motivations et les croyances qui expliquent le comportement des personnages largement implicites. Mais dans le texte ci-dessous, créé par un logiciel d'intelligence artificielle nommé Talespin et développé dans les 
années soixante-dix par James Meehan, les croyances et intentions des personnages sont représentés dans tous leurs détails. Si le programme omettait une seule inférence, il produirait des histoires absurdes :

Il était une fois un ours appelé John qui vivait dans une grotte. John l'ours savait que John était dans sa grotte. Un jour, John a eu très faim. John voulait obtenir du miel. John voulait savoir où il y avait du miel. John aimait Arthur l'oiseau. John voulait qu'Arthur dise à John où il y avait du miel. John était honnête avec Arthur. John avait une bonne relation avec Arthur. John pensait qu'Arthur l'aimait. John pensait qu'Arthur était honnête avec lui. John voulait demander à Arthur de lui dire où il y avait du miel. (Meehan $1980: 200$ )

22 Un critique littéraire dirait probablement que ce texte prend le lecteur pour un idiot. Mais ce n'est pas la production standard de Talespin. Pour chaque histoire que Talespin génère, le programme produit un texte normal et un texte "prolixe». La citation cidessus est la version prolixe. Elle rend explicite la couche de « ce qui va sans dire » qui sous-tend le comportement des personnages. Cette couche doit être reconstruite non seulement par le lecteur, mais aussi par les personnages qui interagissent avec John l'ours.

23 Un aspect important de ce raisonnement tient dans les croyances concernant les croyances des autres personnages : ici John l'ours suppose qu'Arthur l'oiseau est bien disposé envers lui. La formation de ces croyances sur les croyances des autres est indispensable à la réussite de la coopération : si John l'ours se trompe, autrement dit si Arthur l'oiseau n'est pas bien disposé à son égard, l'ensemble de son plan pour obtenir du miel échouera. Dans les récits de coopération, les personnages interprètent correctement les pensées des autres; dans les récits de tromperie ou d'incompréhension, ils forment des représentations erronées. Ce processus d'expliquer les actions d'autrui en construisant leurs états mentaux n'est pas limité à la compréhension des récits, il représente au contraire un mode fondamental du fonctionnement de l'esprit humain, et son domaine d'application comprend les êtres réels autant que les personnages fictifs. Il est connu dans les sciences cognitives comme psychologie populaire («folk psyschology») ou «théorie de l'esprit» («theory of $\left.\operatorname{mind}^{8} »\right)$.

24 Toute personne intéressée par la logique de l'intrigue, tout particulièrement par les manifestations de cette logique dans les fables, les contes de fées, ou la comédie d'erreurs, est consciente de ces effets de miroir. L'influence de la science cognitive sur la narratologie a conduit à un intérêt accru pour ce genre de phénomènes. Lisa Zunshine voit en eux la réponse au titre de son livre Pourquoi nous lisons la fiction (Why We Read Fiction). Selon Zunshine, le plaisir que nous prenons à lire des romans est notre curiosité voyeuriste pour le contenu des pensées des autres. Dans la mesure où la logique de l'intrigue repose sur la construction des croyances, désirs, buts et plans des personnages, elle satisfait ce besoin que nous avons d'exercer notre théorie de l'esprit. Il reste toutefois à voir si le voyeurisme mental explique l'attrait de tout type de fiction, ou s'il fonctionne mieux pour les romans psychologiques comme ceux de Jane Austen ou de Virginia Woolf, que pour la science-fiction, les récits d'aventures, ou le fantastique, genres qui ne mettent pas en scène des raisonnements très compliqués et ne représentent pas les pensées de manière très détaillée. Mais il est indéniable que sans la capacité de construire des représentations de l'esprit des personnages, quel que soit le genre, nous serions incapables de comprendre les histoires, et encore moins de les apprécier. 
25 L'étude des effets de miroir dans la pensée des personnages converge avec mon prochain sujet, l'étude de l'activité mentale du lecteur, parce que ce sont les mêmes mécanismes qui nous permettent de construire le contenu de l'esprit d'autres personnes dans le monde actuel et de comprendre le raisonnement des personnages de fiction. Zunshine (2006) et Herman (2003) pensent que le récit de fiction nous entrâne à construire des effets de miroir plus complexes que dans la vie quotidienne. Selon cette position, la lecture de la fiction représenterait une gymnastique mentale qui accroît le pouvoir de notre cerveau. Sans nier la valeur didactique des récits, je voudrais contester cette thèse.

26 En tant qu'êtres humains, nous sommes très à l'aise avec le calcul de trois niveaux d'effets de miroir : «Il sait que je sais qu'il sait ». Dans certaines rares situations, nous avons même besoin de calculer quatre niveaux, mais cette opération demande un effort considérable. Le récit de fiction peut-il vraiment repousser cette limite? Zunshine (2006: 32-33) affirme qu'un certain passage du roman de Virginia Woolf Mrs Dalloway représente six niveaux, mais son interprétation a été sérieusement contestée par Brian Boyd. J'ai essayé moi-même de diagrammer ce passage, et je n'ai pas pu trouver plus que trois ou quatre niveaux; après quoi les niveaux sont tellement enchevêtrés qu'il est quasiment impossible de déterminer qui pense quoi. Je garde de ce passage un sentiment de chaos qui n'améliore certainement pas ma théorie de l'esprit. Il doit y avoir une bonne raison pour laquelle la biologie nous a doté d'esprits qui calculent facilement trois niveaux: c'est le nombre de niveaux nécessaire pour déceler la tromperie. Par exemple, dans la fable "Le Corbeau et le renard», le renard veut (niveau 1) que le corbeau croie (niveau 2) que le renard désire (niveau 3) l'entendre chanter. Notre capacité de calculer trois niveaux nous aide non seulement à planifier, mais aussi à détecter la tromperie. Notre théorie de l'esprit peut s'étendre à quatre niveaux dans les cas de double tromperie : quelqu'un veut tromper quelqu'un d'autre, mais la future victime identifie correctement cette intention, et fait semblant de tomber dans le piège, afin de tromper le trompeur. Un exemple est l'histoire du coq et du renard analysée par Bruce (1980 ; voir aussi Ryan, 1991) : le renard invite le coq pour le déjeuner avec l'intention de le manger. Le coq fait semblant de croire que le renard est bien disposé envers lui, et lui demande s'il peut amener un ami. Le renard, espérant avoir deux coqs pour le déjeuner, accepte, mais quand le coq arrive chez le renard, il est accompagné d'un chien qui mord le renard et le met en fuite. Le raisonnement du coq peut être représenté de la manière suivante : le coq veut (1) que le renard croie (2) que le coq pense (3) que le renard désire (4) prendre le déjeuner avec lui (plutôt que de faire du coq un déjeuner). Comme le montre cet exemple, le récit peut présenter des cas de quatre niveaux d'intentionnalité, mais je doute sérieusement que les histoires peuvent nous apprendre à calculer des niveaux supplémentaires, car aller au-delà de quatre n'a pas d'utilité pratique. Les personnages de fiction n'auraient donc aucune raison de s'engager dans ce genre de raisonnement. Il est bien sûr toujours possible d'inclure dans un récit des phrases récursives sans fin, comme «Pierre veux demander à Marie de dire à Paul d'aider Jean de convaincre Alice d'épouser Arthur ", mais de telles phrases seront traitées comme une chaîne absurde de mots, et non pas comme une situation importante pour l'intrigue qui demande à être mentalement modélisée par le lecteur. 


\section{L'activité mentale du lecteur}

La question plus générale de l'activité interprétative du lecteur ou spectateur a été explorée aussi bien par la branche littéraire de la narratologie cognitive que par la branche scientifique. L'approche littéraire est le "reader response criticism», une école fondée par Wolfgang Iser qui s'inspire des travaux du philosophe Roman Ingarden sur la phénoménologie de la lecture. Le « reader response criticism » se donne pour but d'explorer les réactions d'un «lecteur modèle " ou "lecteur standard" à des textes littéraires complexes, mais les représentants de cette école ont tendance à se substituer au lecteur modèle, réduisant ainsi leurs travaux à leur interprétation personnelle des textes. Rapidement tombé dans la tradition de la performance de lecture du critique virtuose, le "reader response " s'intéresse plus à construire une théorie esthétique de la signification littéraire qu'à décrire les opérations concrètes qui sous-tendent la construction du sens. Dans les années quatre-vingt-dix, la domination des études littéraires par la théorie culturelle, et l'intérêt naissant pour le contexte historique et social de la littérature a fragmenté le "lecteur modèle » en plusieurs sous-espèces basées sur le genre, la race et la classe, mettant l'accent sur ce qui sépare les lecteurs, au détriment de ce qui les unit. L'idée d'une phénoménologie générale de la lecture est ainsi tombée en désuétude chez les littéraires, mais elle demeure vivante dans les disciplines scientifiques.

Les approches scientifiques s'appuient sur les réactions de sujets autres que l'enquêteur. Il existe essentiellement trois manières de mesurer l'activité mentale associée à la narration. La première, et la plus objective, est celle que j'ai décrite au début de cet article: elle consiste à utiliser la technologie d'imagerie, comme l'IRM, pour enregistrer automatiquement les signaux électriques émis par le cerveau pendant la lecture de textes narratifs. Mais pour pouvoir associer les images IRM à des passages précis, il est nécessaire de contrôler l'écoulement temporel de la lecture en présentant le texte mot à mot sur un écran. Cette procédure crée un mode de lecture si artificiel qu'elle limite considérablement la possibilité de saisir ce qui se passe dans l'esprit du lecteur.

29 La deuxième méthode consiste à créer des expériences qui testent la compréhension et la mémorisation de textes en demandant aux sujets d'accomplir certaines tâches, comme appuyer sur un bouton quand ils détectent une certaine idée. Comme nous l'avons vu, la plupart de ces expériences utilisent des histoires composées spécialement pour l'occasion que personne ne lirait pour leur intérêt narratif intrinsèque ${ }^{9}$. Par exemple, dans une expérience visant à comparer le temps de réaction nécessaire pour déchiffrer des textes cohérents et incohérents, les sujets ont lu ce texte en plusieurs variantes :

Aujourd'hui, Marie avait rendez-vous avec un ami pour le déjeuner. Elle est arrivée tôt au restaurant et a décidé de réserver une table. Puis elle s'est assise et a étudié le menu.

(A) C'était le restaurant préféré de Marie parce qu'il avait de la malbouffe fantastique. Marie adorait tout ce qui était rapide et facile à préparer. Elle mangeait au moins trois fois par semaine chez McDonald. Marie ne surveillait pas son régime et ne voyait aucune raison de manger des aliments nutritifs.

(B) C'était le restaurant préféré de Marie parce qu'il avait des aliments très sains. Marie, obsédée par la santé, était végétarienne depuis dix ans. Son aliment préféré était le chou-fleur. Marie était si stricte sur son régime qu'elle a refusé de manger tout ce qui a était frit ou cuit dans la graisse. 
(C) ...

Marie commanda un cheeseburger et des frites.

(Texte utilisé par O'Brien et al. 1998, cité Gerrig et Egidi 2003 : 50)

30 La troisième méthode est la moins rigoureuse, mais probablement celle dont la narratologie cognitive a le plus à apprendre. Elle consiste à demander aux lecteurs de décrire dans leurs propres termes leur expérience d'une histoire. Victor Nell l'a utilisée de manière très productive dans Lost in a Book, son étude magistrale de l'expérience d'immersion. Des trois méthodes décrites ici, celle-ci est la plus capable de capter ce que Roland Barthes appelle le plaisir du texte, car elle permet d'étudier les réactions personnelles de lecteurs réels à des textes littéraires authentiques - le type de texte qu'on choisit à lire pour sa valeur esthétique et narrative.

31 La narratologie cognitive veut être plus rigoureuse que le «reader response criticism ", mais elle partage la réticence de la critique littéraire pour une approche expérimentale. Ses représentants tendent à s'appuyer sur leur propre lecture du texte, plutôt que sur des données fournies par d'autres lecteurs, parce qu'en travaillant de cette manière, ils peuvent utiliser les textes pour démontrer des idées générales sur la cognition. Contrairement aux praticiens des approches expérimentales, ils s'intéressent moins aux opérations fondamentales du processus de compréhension qu'à un niveau plus élevé de sens qui comprend l'appréciation esthétique, l'impact émotionnel, la dimension symbolique et l'importance existentielle. Il ne s'agit pas là de questions auxquelles on peut répondre par l'expérimentation : leurs réponses, toujours partielles et temporaires, ne peuvent provenir que d'une rencontre personnelle avec les textes narratifs. On revient ainsi à une lecture basée sur l'intuition du chercheur; la principale différence entre le "reader response » d'hier et la narratologie cognitive d'aujourd'hui est que cette dernière est beaucoup mieux informée de la science cognitive.

\section{La narration comme un mode de pensée}

32 La narration comme mode de pensée déplace le centre d'attention de l'esprit qui décode l'histoire à l'esprit qui la conçoit. Cet esprit peut être celui de l'auteur, quand il $\mathrm{y}$ a un texte et un acte de communication, mais pour de nombreux chercheurs une histoire peut être une représentation mentale qui ne s'extériorise pas. Cette conception est particulièrement répandue chez les psychologues et critiques qui conçoivent le soi [self] comme une construction narrative, comme Jerome Bruner et Paul John Eakin, car avoir un soi n'est pas le privilège exclusif des auteurs d'autobiographie.

33 La conception du récit comme mode de pensée recouvre non seulement l'activité de raconter des histoires, elle dirige également l'attention sur l'importance de la communication narrative pour le développement de l'intelligence humaine et la création de relations sociales. Nous touchons ici à la question plus large de la contribution de la faculté narrative à la survie de l'espèce, question favorite des adhérents à l'approche évolutionniste de l'art, tels que Brian Boyd, Dennis Dutton et Joseph Carroll.

34 Contrairement aux études de l'activité mentale du lecteur, les approches du récit comme mode de pensée sont presque entièrement spéculatives. Dans les approches évolutives, la spéculation est inévitable. Comme nous n'avons pas à notre disposition d'exemplaire d'homme de Néandertal ou de Cro-Magnon, il est impossible de comparer 
la compétence narrative de l'humanité au cours des différentes étapes de son évolution culturelle ou biologique, et nous ne pouvons qu'émettre des hypothèses sur le rôle de la narration dans le développement de l'intelligence et l'organisation des sociétés humaines.

35 Les méthodes expérimentales conçues pour mesurer la compréhension des textes narratifs ne fonctionnent pas dans le cas de la production du récit. On ne pourrait pas demander à un conteur de répondre à des questions au milieu d'une performance, ni prendre une IRM d'un romancier en train d'écrire sans sérieusement perturber leur activité mentale. La lecture attentive des textes narratifs est jusqu'à présent la seule façon d'étudier le mode de pensée que nous appelons récit.

36 La nature très spéculative de l'étude de la narration comme mode de pensée a donné libre cours à l'imagination des chercheurs, inspirant un certain nombre de théories qui enflent l'importance cognitive du récit :

37 - Roger Schank (1995) émet l'hypothèse que tous les souvenirs prennent une forme narrative.

- Mark Turner (1996) suggère que l'humanité a inventé le langage pour satisfaire à son besoin de raconter des histoires, au lieu d'avoir commencé à raconter des histoires grâce à l'extension des facultés de communication permises par le langage.

- Jerome Bruner propose trois thèses: (1) Le récit construit la réalité (1991). (2) L'identité est une construction narrative $(2002)^{10}$. (3) La narration nous permet d'acquérir une théorie de l'esprit, et le développement d'une telle théorie est indispensable à la vie communautaire. Il s'ensuit que la faculté narrative est le fondement de l'organisation des sociétés humaines (2002) (Cette idée que c'est par le récit que nous faisons l'acquisition d'une théorie de l'esprit a été plus systématiquement développée par Daniel Hutto sous le titre d' « hypothèse narrative »).

- Pour David Herman, l'expérience humaine n'est pas seulement l'objet de la narration, elle est rendue possible par l'acte même de la narration : « we cannot have a notion of a felt experience without narrative " (nous ne pouvons pas nous représenter ce qu'est une experience vécue sans le récit) (2009:145) et «narrative affords a basis or context for having an experience in the first place » (c'est le récit qui offre en premier lieu une base ou un contexte pour avoir une expérience) (2009:153).

38 La plupart de ces déclarations peuvent être interprétées de deux manières : l'une métaphorique et faible, l'autre littérale et forte. C'est dans le sens littéral qu'elles sont inflationnistes: par exemple, l'idée que le récit construit la réalité est tout à fait acceptable si on l'interprète comme signifiant que le récit donne forme à ce qu'il représente ; mais elle devient contestable si on l'interprète comme signifiant que toutes les perceptions ou les expériences de la réalité ont une forme narrative, ou que la réalité n'existe pas en dehors de la narration.

39 L'une des caractéristiques des théories inflationnistes est qu'elles traitent le récit comme un tout sans distinguer des constituants spécifiques. Selon ces théories, ce serait la possession innée d'une faculté narrative, faculté comparable à l'idée que Chomsky se fait de la compétence linguistique et de la grammaire universelle, qui nous permettrait d'avoir un sens de notre identité, d'acquérir une théorie de l'esprit, de former des souvenirs, ou de saisir le vécu de l'expérience personnelle. Il est typique du discours de l'inflation narrative d'inverser les relations causales, telles que se les représente une vision traditionnelle. Dans cette vision traditionnelle, nous donnons le nom de "récit» à une représentation qui remplit la condition de concerner des 
personnages qui conçoivent des buts, subissent des conflits, ont des émotions, accomplissent des actions, et créent de la sorte des changements dans le monde. Dans la perspective inflationniste, les choses sont inversées : le récit n'est pas le produit de certaines opérations mentales, mais la source de notre capacité d'effectuer ces opérations. Si nous n'avions pas la capacité innée de raconter, nous ne pourrions pas analyser l'expérience vitale en termes d'agents, d'actions, de buts, de plans, de succès, d'échecs, et de changements d'état. David Herman (2009) capture ce point de vue quand il appelle le récit un "outil pour penser» («tool for thinking») et le décrit non pas comme quelque chose que les gens produisent, mais comme quelque chose que les gens «utilisent » pour communiquer certains types d'expérience.

40 Pour formuler plus précisément la question du statut cognitif de la notion de récit, appelons les facultés nécessaires à la construction ou à la compréhension des histoires (a), (b) et (c). Ces variables représentent des opérations telles que: éprouver des émotions, avoir un sens de la séquence chronologique, être en mesure d'inférer des relations causales entre événements et être capable d'expliquer les actions en attribuant à leurs agents des buts et des plans. Le dilemme est le suivant :

Est-ce la possession d'un schéma inné $\mathrm{H}$, un schéma inscrit dans certaines régions du cerveau, qui nous permet d'effectuer (a), (b) ou (c) ? Dans ce cas, il devrait être possible de localiser $\mathrm{H}$ avec une IRM très avancée. La postulation d'un tel schéma représente une approche de haut en bas.

Ou bien est-ce nos facultés innées (a), (b), et (c), que nous avons développées pour résoudre les problèmes de la vie quotidienne, qui nous permettent de communiquer par des histoires?

Dans cette seconde interprétation, le schéma narratif $\mathrm{H}$ n'est pas un outil cognitif autonome qui nous est donné par notre héritage biologique, mais le nom que les narratologues donnent à la convergence des facultés (a), (b) et (c). Cette interprétation, qui procède de bas en haut, nie que le récit exige des facultés mentale spécialisées, je veux dire par là des facultés que nous n'exerçons que quand nous nous produisons ou interprétons des récits. Imaginons que (a) est le raisonnement causal, (b) l'enchaînement temporel, et (c) la théorie de l'esprit : nous utilisons (a) quand nous faisons bouillir de l'eau pour cuire un œuf, (b) lorsque nous planifions notre emploi du temps, et (c) lorsque nous participons à une conversation. Ces activités ne sont pas en elles-mêmes des actes narratifs, même si elles reposent sur des opérations qui sont essentielles à la compétence narrative. Ce n'est que quand (a), (b) et (c) convergent pour produire une représentation mentale qu'on peut parler de récit. Quand les gens perdent la capacité de raconter des histoires, c'est-à-dire, quand ils souffrent de ce que Young et Saver appellent des troubles narratifs (" narrative disorders»), cette perte n'est pas celle d'un modèle global $\mathrm{N}$, elle est due au contraire à une incapacité d'effectuer l'une des opérations nécessaires à la narration. En d'autres termes, les troubles narratifs ne sont pas une condition spécialisée qui n'affecte que la narration, laissant intactes toutes les autres facultés; ils sont causés par des lésions du cerveau qui se traduisent par une perte de facultés individuelles, telles que la mémoire, la capacité de distinguer les faits de l'invention, ou la capacité d'organiser l'information en séquences temporelles. Les personnes qui souffrent de troubles narratifs ne sont pas dysfonctionnelles parce qu'elles ont perdu leur compétence narrative; elles perdent leur compétence narrative parce qu'elles sont incapables d'effectuer l'une ou l'autre des opérations cognitives qui permettent à la fois de vivre la vie et de la raconter. 
Un compromis entre les deux interprétations consiste à les considérer comme reliées l'une à l'autre par une boucle rétroactive. Il semble raisonnable d'affirmer que les histoires exercent et par conséquent renforcent notre capacité d'effectuer (a), (b) et (c). Les récits racontent la résolution de problèmes, le succès et l'échec, l'interaction dans la vie entre le hasard et l'action planifiée, le contenu de la pensée des autres; et ils pourraient très bien nous aider à interpréter la vie selon ces catégories. Mais il me semble que quelque chose doit venir en premier entre l'œuf et la poule. Je ne peux pas imaginer comment un enfant incapable de comprendre les notions de causalité ou de temporalité, et n'étant pas conscient que les autres personnes sont capables de penser, pourrait acquérir ce genre de connaissances en écoutant des histoires. Sur le plan de l'espèce, je ne vois pas comment les humains auraient pu commencer à raconter des histoires s'ils n'avaient pas une capacité de raisonnement causale déjà bien développée. Si la narration repose sur des opérations mentales aussi essentielles à la survie de l'espèce que la compréhension de la causalité et l'attribution de pensées aux autres, il y a d'innombrables autres situations dans la vie qui nécessitent de telles capacités. Dans un modèle de boucle rétroactive, le récit renforce ce que la vie nous enseigne, parce que le récit, qu'il soit factuel ou fictif, est une représentation de la vie. (Voir figure 1)

\section{Qu'est-ce que la narratologie cognitive?}

45 En conclusion, je voudrais revenir à la question: que fait-on quand on fait de la narratologie cognitive? La procédure la plus évidente consiste à emprunter des concepts aux disciplines cognitives, et à les appliquer de haut en bas au récit. Ces concepts ont tendance à provenir des domaines les plus spéculatifs des disciplines cognitives, tels que la psychologie sociale et la philosophie de l'esprit, plutôt que des approches expérimentales. Le plus populaire de ces imports est la notion déjà discutée de théorie de l'esprit. Herman $(2007,2010)$ propose cinq candidats supplémentaires pour l'application de haut en bas : la théorie du positionnement (" positioning theory ", comment les narrateurs se présentent à leur audience et comment les personnages, $y$ compris le narrateur, imposent des rôles aux autres personnages); l'incorporation ("embodiment », l'idée que l'esprit est inséparable du corps, et que la pensée est profondément affectée par cette relation); la nature «distribuée» de l'esprit (" distributed cognition », l'idée que les objets externes qui stimulent la pensée font partie intégrale de l'esprit); le discours de l'émotion et l'« émotionologie » (théories scientifiques de la nature des émotions); et qualia (le terme philosophique pour la qualité spécifique et intimement personnelle de l'expérience). Un autre exemple de méthode de haut en bas est l'approche d'Alan Palmer, qui emprunte à la psychologie sociale l'idée d'un esprit collectif ou interpersonnel pour enquêter sur les manifestations de l'opinion publique dans les romans, par exemple l'esprit de la ville de Middlemarch dans le roman éponyme de George Eliot. Dans le meilleur des cas, les idées importées des sciences cognitives attirent l'attention sur des phénomènes jusqu'à présent négligés et fournissent ainsi de nouvelles perspectives sur le récit (c'est le cas pour Palmer ou pour l'application par Herman de la "positioning theory »); dans le pire des cas il existe peu de liens entre le concept adopté et les aspects du texte narratif qu'il est censé révéler. Comme elles ne prescrivent pas de méthode analytique précise, les notions empruntées aux disciplines cognitives laissent une grande liberté d'interprétation, et les chercheurs trouvent presque inévitablement ce qu'ils recherchent. Mais la méthode de haut en bas ne fournit pas de solutions aux dilemmes 
des disciplines cognitives : par exemple, ce n'est pas en analysant des récits qu'on peut choisir entre les trois explications de la théorie de l'esprit proposées jusqu'à présent : la "théorie -théorie »; la simulation mentale, ou l'hypothèse narrative de Hutto ${ }^{11}$. Ce n'est de même pas en étudiant le récit qu'on peut choisir entre les solutions physicaliste et dualiste du problème corps-esprit: même si la position physicaliste domine actuellement l'opinion des chercheurs, les récits reflètent généralement le point de vue de leur époque, et de nombreuses histoires, tout particulièrement les histoires d'inspiration religieuse, adoptent une position explicitement dualiste ${ }^{12}$. Il faudrait une lecture déconstructionniste pour faire dire à ces récits ce qu'ils nient ouvertement. Mais il est incontestable que certaines théories sont plus productives que d'autres en narratologie cognitive quelle que soit leur valeur scientifique - je pense à l'enactivisme (Caracciolo 2013) par rapport au cognitivisme ${ }^{13}$ - parce qu'elles inspirent de nouvelles manières d'aborder la narration, et parce qu'elles jouissent du prestige de représenter l'avant-garde en matière cognitive.

46 Une manière plus souple pour la narratologie de s'inspirer des disciplines cognitives serait de pratiquer une méthode de convergence. Au lieu de chercher à vérifier à tout prix dans les textes les concepts mis à la mode par les disciplines cognitives, cette méthode consisterait à citer la recherche scientifique à l'appui de thèses développées plus ou moins indépendamment. La narratologie rechercherait ainsi la bénédiction de la science, mais sans se laisser réduire en esclavage. Anežka Kuzmičová utilise par exemple l'article de Zacks et Speer discuté ci-dessus (avec un grand nombre d'autres travaux) pour défendre l'idée que la représentation d'un corps en mouvement crée une expérience d'immersion spatiale beaucoup plus intense qu'une représentation limitée à la description d'objets statiques. Elle démontre de façon convaincante que les corrélations détectées par Zacks et Speer entre la lecture d'une description verbale de gestes et la performance actuelle de ces gestes signifient que la simulation mentale par le lecteur d'un corps en mouvement conjure un sens intensifié de la présence de l'environnement spatial décrit dans le texte. La méthode de convergence peut également être utilisée pour situer les problèmes narratologiques dans un contexte plus large. Je pense ici à l'examen des approches cognitives de l'émotion qu'on trouve dans le livre de Suzanne Keen sur l'empathie narrative et dans celui de Torben Grodal sur le pouvoir émotionnel du cinéma. Le but de ces aperçus n'est pas d'en tirer une méthode d'analyse, mais de situer la recherche de l'auteur dans un vaste panorama interdisciplinaire. De même, pour le lecteur de Guillemette Bolens sur la poétique des gestes en littérature, le bénéfice est double, puisque le lecteur apprend à lire les textes, tout en s'informant sur le discours scientifique relatif aux gestes.

47 Dans toutes les approches esquissées ci-dessus, la narratologie se sert de la science cognitive ; mais peut-elle rendre la pareille en faisant une contribution substantielle à la science cognitive, comme le voudrait David Herman (2013 : ix) ? Si l'on s'en tient à un niveau très élémentaire elle en est certes capable. La science expérimentale a besoin d'hypothèses à vérifier; quand elle s'intéresse à l'activité mentale suscitée par la narration, où trouvera-t-elle ces hypothèses, si ce n'est dans l'étude du récit, ce qui veut dire, en narratologie? Quand les psychologues conçoivent des expériences pour examiner des problèmes tels que comment les histoires créent le suspense, comment les lecteurs traitent les mondes logiquemnt inconsistants, ou ce que signifie être immergé dans un monde narratif, ils peuvent soit avoir pris conscience de ces problèmes grâce à la narratologie, soit les avoir identifiés spontanément, mais dans ce 
second cas, on peut dire qu'ils pensent comme des narratologues. Jean-Marie Schaeffer (2010: 229-30) doute cependant qu'une collaboration plus étroite puisse avoir lieu entre les approches expérimentales et narratologique, parce que la science cognitive et la narratologie poursuivent des objectifs différents: la première vise à la validité descriptive, alors que la seconde vise à la valeur explicative. Pour un chercheur en neurologie, établir une cartographie de l'intelligence narrative, ce qui veut dire localiser les régions du cerveau responsables de la compréhension et de la production du récit, comme l'a fait Raymond Mar, constitue une fin en soi ; pour la narratologie cognitive, au contraire, cette cartographie ne présente d'intérêt que dans la mesure où elle permet de mieux comprendre le fonctionnement de l'intelligence narrative. Chacune des deux approches ambitionne de faire des prédictions précises, mais l'approche narratologique est théoriquement plus ambitieuse, plus ouverte à la spéculation et aux généralisations, et fait une plus grande part à la créativité du chercheur.

48 Il y aura un feed-back entre les sciences cognitives dures et la narratologie quand des expériences scientifiques telles que l'imagerie du cerveau deviendront capables d'injecter de nouvelles idées en narratologie, plutôt que de vérifier des idées qui vont de soi. Mais il faudrait d'abord que la narratologie développe une idée plus précise de ce qui constituerait pour elle des résultats scientifiques intéressants. Un exemple de ce qui est à mon avis un résultat intéressant est une expérience par IMR conduite par Anna Abraham et ses collègues de l'Institut Max Planck à Leipzig (Abraham et al., 2008). Dans cette expérience, les sujets ont été invités à imaginer des scénarios comprenant des personnages réels et imaginaires (par exemple, rencontrer George Bush vs. rencontrer Cendrillon), et une IRM de leur cerveau a été prise dans chaque cas. Il s'est avéré que différentes régions du cerveau ont été activées pour les personnages réels et fictifs : pour Cendrillon, une région qui correspond à des faits établis ; pour George Bush, une région plus propice à la révision. Cette différence peut apparaître à première vue surprenante, mais elle confirme ce que les théoriciens de la fiction nous disent (Doležel 1998 ; Ryan, 1991). Dans la mesure où les personnages fictifs sont créés par des textes, ils sont le produit d'un corpus limité d'information. Avec des personnages de la vie réelle, par contre, le corpus est ouvert, car il est toujours possible d'obtenir de nouvelles informations, qui conduiront à la révision de notre représentation. En outre, nous savons parfaitement que nous ne pourrons jamais rencontrer Cendrillon, puisqu'elle n'existe pas, mais nous pouvons imaginer des circonstances qui mèneront à une rencontre avec George Bush. D'un point de vue narratologique, la démonstration neurologique que le cerveau est capable de distinguer George Bush, personnage réel, de Cendrillon, personnage fictionnel, ne fait que vérifier ce que le bon sens nous dit. Par contre, les résultats d'Abraham suggèrent quelque chose qui ne va pas de soi : à savoir que les vérités concernant le monde réel sont plus problématiques que les "vérités fictives ». En d'autres termes, nous pouvons faire toute confiance aux faits allégués par un roman au sujet d'un monde fictif, alors que les faits allégués par les historiens relatifs au monde réel peuvent toujours être mis en doute.

49 Dans l'attente d'une véritable boucle rétroactive entre les disciplines cognitives et la narratologie, comment faut-il penser le lien entre le récit et l'esprit, soit producteur, soit récepteur? Ma réponse est très simple : en posant des questions telles que :

50 - Qu'est-ce qui rend une histoire racontable («tellable»), et quelles sont les caractéristiques qui peuvent constituer la raison d'être d'une histoire? 
- Quels sont les dispositifs de présentation qui attirent l'attention de l'auditoire?

- Quelle est la nature des émotions produites par les récits, et comment se fait-il que nous éprouvions du plaisir même quand les récits racontent des événements tragiques?

- Que signifie être immergé dans une histoire, et quelles sont les caractéristiques qui favorisent ou inhibent l'immersion?

- Comment définir la fiction, et pourquoi sommes-nous passionnés par le sort de personnages qui n'ont jamais existé?

- Plus généralement, quel est le rôle de l'invention et du raisonnement contrefactuel dans la vie de l'esprit ou dans l'évolution de l'intelligence humaine?

- Par quels mécanismes les lecteurs sont-ils capables de construire une image cohérente des mondes narratifs sur la base d'une information très partielle?

- Comment les lecteurs traitent-ils des mondes narratifs qui contiennent des contradictions?

- Quelle est la nature d'une expérience esthétique du récit?

- Quels sont les thèmes et techniques narratives qu'on retrouve dans toutes les cultures et périodes historiques (cf. Hogan 2010) ? Peut-on élaborer un modèle universel de l'intrigue?

51 Si la narratologie cognitive existe véritablement, elle consiste bien moins à emprunter des concepts tout faits aux sciences cognitives et à les appliquer aux textes de haut en bas, qu'à faire confiance à la capacité de notre intelligence de comprendre comment elle crée, décode et utilise des histoires. En d'autres termes, la narratologie cognitive doit faire confiance à la capacité de l'intelligence de se comprendre elle-même. Quand David Herman identifie son objet comme "les relations de la narration et de l'esprit " ( " the nexus of narrative and mind»), il définit ces relations comme « les aspects de la pratique de raconter des histoires qui mettent en jeu l'intelligence» («the mindrelevant aspects of storytelling practices») (2009: 140). Cette formule peut être interprétée de deux manières: (1) Au niveau du contenu des histoires, comme la manière dont la narration représente tout ce qui touche à l'esprit (les pensées des personnages, leurs relations au monde extérieur, leur expérience intime, leur psychologie, voire l'expression de leur état mental par les mouvements de leur corps). Il existe ainsi des aspects du récit qui se rapportent à l'esprit et d'autres qui ne le font pas. Pour qu'il y ait une boucle rétroactive entre la narratologie et la science cognitive les données fournies par les récits et les analyses de ces données par les narratologues devraient être utiles à la science cognitive. Cette question est loin d'être résolue ${ }^{14}$. (2) Au niveau de la création/réception, la formule d'Herman peut être interprétée comme la manière dont l'esprit met en intrigue l'expérience du monde et construit des mondes à partir des récits ${ }^{15}$. Dans cette interprétation, il est impossible de distinguer les aspects de la narration qui mettent en jeu l'esprit des aspects qui ne le font pas : tout dans la narration parle de l'intelligence qui la crée et de l'intelligence qui l'interprète. On peut dès lors faire de la narratologie cognitive sans le savoir, tout comme Monsieur Jourdain faisait de la prose, parce qu'étudier le récit, c'est étudier le fonctionnement de l'esprit humain dans l'une de ses manifestations les plus fondamentales, les plus universelles, et les plus complexes. 


\section{BIBLIOGRAPHY}

Abraham, Anna, D. Yves von Cramon, and Ricarda I. Schubotz. "Meeting George Bush versus Meeting Cinderella: The Neural Response When Telling Apart What is Real from What is Fictional in the Context of Our Reality." Journal of Cognitive Neuroscience 20.6 (2008): 965-976.

Banfield, Ann. Unspeakable Sentences: Narration and Representation in the Language of Fiction. Boston: Routledge \& Kegan Paul, 1982.

Bernaerts, Las, Dirk de Geest, Luc Herman and Baert Vervaek, eds. Stories and Minds: Cognitive Approaches to Literary Narrative. Lincoln: University of Nebraska Press, 2013.

Bolens, Guillemette. Le Style des gestes. Corporéité et kinésie dans le récit littéraire. Lausanne : Éditions BHMS, 2008.

Bor, Daniel. "The Mechanics of Mind Reading." Scientific American Mind July/August 2010: 52-7. Borges, Jorge Luis. « Funès ou la mémoire. » Fictions, traduit par P. Verdevoye, Ibarra et Roger Caillois. Paris : Gallimard, 1983, collection Folio. 109-118.

Bortolussi, Marisa, and Peter Dixon. "Minding the Text: Memories for Literary Narrative." Bernaerts et al., 23-37.

Boyd, Brian. "Fiction and Theory of Mind." Philosophy and Literature 30 (2006):571-81.

---. On the Origin of Stories. Evolution, Cognition and Fiction. Cambridge, Mass.: Harvard University Press, 2009.

Bruce, Bertram. "Analysis of Interacting Plans as a Guide to the Understanding of Story Structure.” Poetics 9 (1980):195-311.

Bruner, Jerome. “The Narrative Construction of Reality.” Critical Inquiry 18 (1991): 1-18.

---. Making Stories: Law, Literature, Life. Cambridge, Mass.: Harvard University Press, 2002.

Carroll, Joseph. “An Evolutionary Paradigm for Literary Study.” Style 42, 2-3 (2008): 103-35.

Caracciolo, Marco. "Blind Reading: Toward an Enactivist Theory of the Reader's Imagination. "Bernaerts et al., 81-105.

Cohen, Patricia. "Next Big Thing in English: Knowing They Know That You Know.” New York Times, April 1 2010. http://www.nytimes.com/2010/04/01/books/01lit.html

Cohn, Dorrit. Transparent Minds: Narrative Modes for Presenting Consciousness in Fiction. Princeton: Princeton University Press, 1978.

---. The Distinction of Fiction. Baltimore: Johns Hopkins University Press, 1999

Currie, Gregory. “On Not Learning About the Mind from Literature.” 2011. https://

www.academia.edu/1686169/On_not_learning_about_the_mind_from_literature

Doležel, Lubomír. Heterocosmica: Fiction and Possible Worlds. Baltimore: Johns Hopkins University Press, 1998.

Dutton, Denis. The Art Instinct. New York: Bloomsbury Press, 2009.

Eakin, Paul John. "What We Are Reading When We Are Reading Autobiography." Narrative 12.2 (2004): 121-32. 
Emmott, Catherine. Narrative Comprehension: A Discourse Perspective. Oxford: Oxford University Press, 1997.

Everding, Gerry. "Readers Build Vivid Mental Simulations of Narrative Situations, Brain Scans Suggest." Record (Washington University). February 12 2009. http://news.wustl.edu/news/Pages/ 13383.aspx

Gerrig, Richard J., and Giovanna Egidi. "Cognitive Psychological Foundations of Narrative Experience." Narrative Theory and the Cognitive Sciences. Ed. David Herman. Stanford, Ca: CLSI Publications, 2003. 33-55.

Goldstein. Philip. "Reader Response Theory and Criticism.” The Johns Hopkins Guide to Literary Theory and Criticism, second edition. Eds. Michael Groden, Martin Kreiswirth and Imre Szeman. Baltimore: Johns Hopkins University Press, 2005. 793-97.

Grodal, Torben. Moving Pictures: A New Theory of Film Genres, Feelings, and Cognition. Oxford: Clarendon Press, 1997.

Herman, David. "Regrounding Narratology: The Study of Narratively Organized Systems for Thinking." What is Narratology? Questions and Answers Regarding the Status of a Theory. Eds. Tom Kindt and Hans-Harald Mueller. Berlin: Walter de Gruyter, 2003. 303-32.

---. "Storytelling and the Sciences of Mind: Cognitive Narratology, Discursive Psychology, and Narratives in Face-to-Face Interaction." Narrative 15.3 (2007): 306-34.

---. "Cognitive Grammar and Focalization Theory." Point of View, Perspective and Focalization. Eds. Peter Hühn, Wolf Schmid and Joerg Schönert. Berlin: Walter de Gruyter, 2009. 119-42.

---. Basic Elements of Narrative. Malden, MA: Wiley-Blackwell, 2009.

---. “Afterword: Narrative and Minds: Directions for Inquiry. Bernaerts et al., 199-209.

---."Introduction." The Emergence of Mind, ed. David Herman. Lincoln: University of Nebraska Press, 2011. 1-40.

---. Storytelling and the Sciences of Mind. Cambridge, MA: MIT Press, 2013.

Hofstadter, Douglas. I am a Strange Loop. New York: Basic Books, 2007.

Hogan, Patrick Colm. “Literary Universals.” Zunshine 2010, 37-60.

Hutto, Daniel D. Folk Psychological Narratives. Cambridge, Mass,: MIT Press, 2008.

Hutto, Daniel D. “The Narrative Practice Hypothesis: Origins and Applications of Folk Psychology." Narrative and Understanding Persons. Ed. Daniel D. Hutto. Cambridge: Cambridge University Press, 2007. 43-68.

Ingarden, Roman. The Literary Work of Art. Trans. George Grabowicz. Evanston: Northwestern University Press, 1973.

Iser, Wolfgang. The Act of Reading: A Theory of Aesthetic Response. Baltimore: Johns Hopkins University Press, 1978.

Keen, Suzanne. “A Theory of Narrative Empathy.” Narrative 14.3 (2006):207-36.

Kintsch, Walter, and Teun A. van Dijk. "Toward a Model of Text Comprehension and Production." Psychological Review 85.5 (1978): 363-94.

Kuzmičová, Anežka. "Presence in the Reading of Literary Narrative: A Case for Motor Enactment." Semiotica 189 (1/4): 23-48. 
Lakoff, George, and Mark Johnson. Metaphors We Live By. Chicago: University of Chicago Press, 1980.

Mandler, Jean, and Nancy Johnson. "Remembrance of Things Parsed." Cognitive Psychology 9 (1977): 111-51.

Mar, Raymond. "The Neuropsychology of Narrative: Story Comprehension, Story Production and their Interrelation." Neuropsychologia 42 (2004):1414-1434.

Meehan, Jim. “Tale-Spin.” Inside Computer Understanding. Ed. Roger Schank. Hillsdale, N.J: Lawrence Erlbaum, 1981. 197-225.

Nell, Victor. Lost in a Book: The Psychology of Reading For Pleasure. New Haven: Yale University Press, 1988.

Oatley, Keith. "Why Fiction May Be Twice as True as Fact: Fiction as Cognitive and Emotional Simulation." Review of General Psychology 3.2 (1999): 101-7.

O’Brien, E.J., M.L. Rizzella, J.E. Albrecht and J.G. Halleran. “Updating a Situation Model: A Memory-Based Text Processing View." Journal of Experimental Psychology: Learning, Memory and Cognition 24 (1998): 1200-10.

Palmer, Alan. Fictional Minds. Lincoln: University of Nebraska Press, 2004.

---. “Social Minds." Style 45.2 (2011),196-240.

Rumelhard, David. "Notes on a Schema for Stories." Representations and Understanding: Studies in Cognitive Science. Eds D.G. Bobrow and M. Collins. New York: Academic Press, 1975.

Ryan, Marie-Laure. Possible Worlds, Artificial Intelligence and Narrative Theory. Bloomington: University of Indiana Press, 1991.

---. Narrative as Virtual Reality: Immersion and Interactivity in Literature and Electronic Media. Baltimore: Johns Hopkins University Press, 2001.

Schank, Roger, and Robert P. Abelson. "Knowledge and Memory: The Real Story." Advances in Social Cognition, vol. 7: Knowledge and Memory: The Real Story. Ed. Robert S. Wyer. Hillsdale, N.J.: Lawrence Erlbaum, 1995. 1-85.

Schaeffer, Jean-Marie. "Le Traitement cognitif de la narration." Narratologies Contemporaines. Eds John Pier and Francis Berthelot. Paris : Editions des archives contemporaines, 2010. 215-32.

Speer, Nicole K., Jeremy R. Reynolds, Kheena M. Swallow, and Jeffrey M. Zacks. "Reading Stories Activates Neural Representations of Perceptual and Motor Experiences." Psychological Science 20 (2009): 989-999.

Starr, G. Gabrielle. “Multisensory Imagery.” Zunshine 2010, 275-91.

Stein, Nancy, and Tom Trabasso. "What's in a Story? An Approach to Comprehension and Instruction." Advances in Instructional Psychology, vol 2. Ed. Robert Glaser. Hillsdale, N.J: Lawrence Erlbaum, 1982.

Strawson, Galen. “Against Narrativity.” Ratio 17 (December 2004): 428-72.

Turner, Mark. The Literary Mind. Oxford: Oxford University Press, 1996.

Young, Kay, and Jeffrey L. Saver. “The Neurology of Narrative.” Substance 30 (2001): 72-84.

Zunshine, Lisa. Why We Read Fiction. Theory of Mind and the Novel. Columbus: The Ohio State University Press, 2006.

---, ed. Introduction to Cognitive Cultural Studies. Baltimore: Johns Hopkins University Press, 2010. 
Zwaan, Rolf. "Situation Model.” The Routledge Encyclopedia of Narrative Theory. Eds. David Herman, Manfred Jahn and Marie-Laure Ryan. London: Routledge, 2005. 534-36.

\section{NOTES}

1. J'emploie «esprit " comme l'équivalent français du mot anglais «mind " pour signifier l'ensemble de nos facultés mentales, ou le fonctionnement du cerveau, bien que je sois consciente qu' « esprit » présente des connotations différentes de «mind» de par son association avec «spirituel ». Pour cette raison j'emploierai aussi d'autres termes, comme intelligence narrative, pour parler des facultés mentales qui permettent aux humains de raconter et de comprendre des histoires.

2. Dans cet article j'emploie souvent « lecteur » pour designer la réception du récit quel que soit son médium. Lecteur peut ainsi tenir lieu de spectateur ou même de joueur dans le cas des jeux vidéo.

3. En fait Speer et ses collègues ont utilisé l'IRMf, imagerie par résonance magnétique fonctionnelle. Tout au long de cet article, je vais utiliser l'IRM pour désigner tout type de technique d'imagerie du cerveau, car les questions technologiques ne sont pas ma préoccupation.

4. L'existence d'un type spécial de neurones peut être vérifiée expérimentalement chez les chimpanzés mais pas chez les humains car de telles expériences seraient trop envahissantes. Ce n'est qu'indirectement qu'on peut étudier le fonctionnement des neurones miroirs chez les humains, et ces études sont loin d'entraîner l'unanimité sur l'existence d'un tel système. Voir l'article «Mirror neurons» sur Wikipedia. http://en.wikipedia.org/wiki/ Mirror_neuron\#In_humans

5. Selon un article paru dans Scientific American Mind (Bor 2010), l'IRM peut détecter si le sujet pense à l'un ou l'autre de deux sujets présélectionnés : le tennis ou la maison. On a demandé aux sujets de penser tennis quand ils voulaient répondre « oui » à une question, et de penser maison pour répondre «non ». C'est là un premier pas dans la lecture des pensées.

6. Cela ne veut pas dire que la spéculation soit absente des disciplines expérimentales; l'interprétation des résultats est toujours spéculative.

7. Par contre je ne suis pas Herman jusqu'au bout quand il déclare que postuler une « intériorité » contenant des pensées secrètes relève d'un dualisme cartésien entre l'esprit et le corps, dualisme généralement contesté par la science cognitive et la philosophie contemporaine. Herman va jusqu'à rejeter l'idée d'un « exceptionalisme » qui accorderait à la fiction une capacité unique de représenter la vie de l'esprit. Si l'intériorité est un mythe, si l'esprit se manifeste dans son mouvement vers le monde, il n'y a pas de pensées secrètes auxquelles seuls les narrateurs omniscients peuvent accéder. L'argument d'Herman rend l'activité mentale d'autrui aussi accessible et représentable dans les récits factuels que fictionnels, conclusion à mon avis inacceptable.

8. Pour certains chercheurs, comme par exemple Daniel Hutto, ces deux étiquettes diffèrent dans leurs implications théoriques, mais dans le cadre de cet article elles peuvent être considérées comme synonymes.

9. Selon Cohen (2010: 3), un projet est en cours à Yale sous la direction de Michael Holquist qui utilise l'IRM pour tester les réactions des lecteurs de textes littéraires authentiques.

10. Cette affirmation a été attaquée dans un célèbre article de Galen Strawson.

11. Les narratologues comme Herman (2009) et Palmer (2011) ont tendance à favoriser l'explication de Hutto, probablement parce qu'elle renforce l'importance de la narration.

12. Une manière de montrer que le récit prouve la corporéité de l'esprit, quelle que soit la façon dont il conçoit le problème corps-esprit, est d'invoquer la présence dans un texte de métaphores conventionnelles qui utilisent le corps comme norme implicite de référence: par exemple, les 
métaphores qui s'appuient sur un contraste entre le haut et le bas, présupposant, selon Lakoff et Johnson, la position verticale du corps. Mais ces métaphores sont une caractéristique générale du langage et non pas une caractéristique spécifique de la narration. Elles sont probablement aussi fréquentes dans le discours non-narratif que dans les récits.

13. L'enactivisme, théorie inspirée par la phénoménologie de la perception, tente de saisir l'esprit dans sa relation avec le monde; le cognitivisme perçoit l'esprit sur le modèle d'un ordinateur, comme un système de décisions logiques.

14. Dans un exposé très controversé, Gregory Currie met en doute la capacité de la littérature de nous apprendre quelque chose sur l'esprit (« On Not Learning About the Mind from Literature »). Le psychologue Keith Oatley maintient par contre l'idée que les auteurs de fiction ont une vision privilégiée de la nature de l'esprit, vision qu'on peut attribuer soit au génie soit au don d'observation.

15. Herman (2013, passim) exprime ces deux formes d'activité mentale par un élégant jeu de mots malheureusement intraduisible en français : «Storying the world » et « Worlding the story ».

\section{ABSTRACTS}

Cognitive approaches have been widely hailed as "the next big thing" in the beleaguered field of literary studies. But despite a widespread interest for what narrative means for the life of the mind, the significance of the concrete contributions of the so-called cognitive sciences to narratology is far from enjoying broad acknowledgment. In this paper I propose a critical examination of the various approaches to narrative that can be called "cognitive", from hard scientific studies based on brain scanning, to psychological experiments that test the reaction of subjects to simple texts composed specially for the occasion, to the import of concepts from the more speculative disciplines of the cognitive sciences, such as social psychology or philosophy of mind. I distinguish three areas of investigation for cognitive narratology: (1) the minds of characters ; (2) the mental activity of the reader (spectator, player, etc) ; (3) narrative as a way of thinking. Against the tendency to conceive cognitive narratology as a matter of borrowing readymade concepts from the cognitive sciences and applying them top-down to texts, I argue in favor of trusting the ability of our own minds to figure out how the mind creates, decodes and uses stories.

Les approches cognitives ont été généralement saluées comme la vague de l'avenir pour la narratologie. Mais en dépit d'un vif intérêt pour ce que le récit révèle de la vie de l'esprit, l'importance des contributions concrètes des sciences dites cognitives à la narratologie est loin d'entraîner l'unanimité. Dans cet article, je propose un examen critique des différentes approches cognitives de la narration. Ces approches vont des études basées sur l'imagerie cérébrale, aux expériences psychologiques qui testent la réaction de sujets à des textes simples composées spécialement pour l'occasion, à l'importation de concepts développés par des disciplines spéculatives, comme la psychologie sociale ou la philosophie de l'esprit. Je distingue trois domaines d'investigation pour la narratologie cognitive : (1) l'esprit des personnages ; (2) l'activité mentale du lecteur (spectateur, joueur, etc.); (3) le récit comme manifestation d'une manière de penser. À la tendance de concevoir la narratologie cognitive comme l'application de haut en bas de concepts empruntés aux sciences cognitives, j'oppose une méthode procédant de 
bas en haut, faisant confiance à la capacité intuitive de notre esprit de comprendre comment il crée, décode et utilise des histoires.

\section{INDEX}

Keywords: cognitive narratology, brain scanning, experimental approaches on narratives, borrowing concepts from cognitive sciences, minds of characters, narrative as way of thinking, mental activity of readers

Mots-clés: narratologie cognitive, imagerie cérébrale, approches expérimentales du récit, emprunts de concepts aux sciences cognitives, esprit des personnages, récit comme manière de penser, activité mentale des lecteurs 